Purdue University

Purdue e-Pubs

ASEE IL-IN Section Conference

\title{
Tackling mathematical difficulties in transport theory education: insight from semiotics
}

Abdel-Rahman Hassan

Purdue University

Follow this and additional works at: https://docs.lib.purdue.edu/aseeil-insectionconference

Hassan, Abdel-Rahman, "Tackling mathematical difficulties in transport theory education: insight from semiotics" (2018). ASEE ILIN Section Conference. 8.

https://docs.lib.purdue.edu/aseeil-insectionconference/2018/innov/8

This document has been made available through Purdue e-Pubs, a service of the Purdue University Libraries. Please contact epubs@purdue.edu for additional information. 


\title{
Tackling mathematical difficulties in transport theory education: insight from semiotics
}

\author{
Abdel-Rahman Hassan \\ Weldon School of Biomedical Engineering, Purdue University, West Lafayette, IN
}

Transport theory involves the study of phenomena most relevant to engineering such as fluid mechanics, heat transfer, and diffusion. These phenomena appear in almost every engineering artifact, from drug delivery to the nuclear reactor. In several disciplines, core courses are dedicated to this science. I have studied and taught transport theory in several engineering disciplines: nuclear engineering, materials science, and biomedical engineering. Here, I reflect on this experience and describe a source of difficulty that runs throughout that experience.

In this paper, I propose a theory of mathematical difficulty for transport courses, an ideal example of engineering courses that rely heavily on mathematical analysis. In section 2, mathematical difficulty is analyzed using the theory of semiotics. I relate this difficulty to the curricular plan in engineering schools and point out its ramifications on learning. I, then, introduce a strategy to eradicate the source of difficulty in section 3. Finally, in section 4, I draw general features of pedagogy to address the source of difficulty and to authentically integrate mathematical knowledge from prior stages into transport courses or theory courses in general.

\section{Motivation}

In a recent casual discussion between me and a junior student, he was looking back at what he learned in a transport course - here is a student with a metacognitive ability. After a bit of thinking, he highlighted the material derivative, an essential concept in transport theory. Although he could identify its criticality to his learned knowledge, he could not interpret it. For him, it was a valuable entity petrified in a mathematical form with a name tag on it. I offered an explanation as to why it is necessary and what it means-I said if you want to know my velocity and the ground under my feet is moving, you would have to take that movement into account; if a property is carried by something else, its transport and its carrier's transport both contribute to its balance. Despite the parable's conciseness, he appeared to be satisfied with that explanation. In another occasion, students showed their confusion between the (partial) time derivative and the material derivative; they had an 'aha' moment when learning their physical significance.

Knowledge within science and engineering consists of elements or information and a structure that relates them, theory and methods. Mathematics provides an excellent structure to information within the body of knowledge of engineering and several sciences. Further, mathematical analysis is indispensable to the coherence of scientific knowledge and its application. We cannot, for instance, provide coherent and useful statements on transport phenomena without the structure provided by vector calculus. Recent trends have called for the minimization of mathematical content in engineering education on the basis that alternative 'tools,' such as MatLab, can take the role. The job of mathematics, I insist, is not only to provide a means of calculating for end resultsa widespread belief - but also that it provides the logical structure and the means whereby information can make sense in relation to each other. The belief is a result of the confusion between laborious mathematical techniques or derivations, and mathematical analysis in its higher level, as 
a means of formal abstraction.

Comforting students who initially struggle with mathematics and minimizing the mathematical component assumes an 'entity' theory of ability: that not everybody can be good in mathematics, or in using mathematics as for engineering purposes; as opposed to an incremental or malleable theory of ability [1]. This educational policy has been shown to 'lock [such] students in long-term low achievement" [2]. Apart from being the logical language, as such, for the whole history of modern science, mathematics underlies transferable development of analytical and problemsolving skills within the essential development of an engineer. It teaches students how to abstract reality, reduce it, define the limits in dealing with it, and to systematically optimize their strategies. Needless to say, this reflects the first of the ABET criteria.

A common strategy to 'get done with' mathematics is the drilling paradigm where students are taught repeatedly how to solve a particular mathematically-formulated problem. The following quote best highlights the shortcomings of this approach. "Perhaps the worst consequence of the drilling paradigm is that students and adults have no ability to use the math they learned in school outside of the boxes within which they were drilled. The value of math is in its predictive powers. You combine math with economics, or math with biology, or math with physics, even with law [...] etc., and suddenly you can predict the future. But these uses of math require extremely strong fundamentals, which most people were never taught." (from 'What's wrong with Math education in the US?', slate.com). In other words, it precludes the transferability of mathematics. Instead of calling for reduction in mathematical content, we should amend our perspectives on mathematics and consequently the way it is taught and applied in engineering curricula [2]. Given the current discourse and pedagogy, it is only logical that the public impression is negative to the extent of a mathematics education crisis [3].

\section{Analyzing a cause of difficulty}

It is proposed that a problem of engineering theory education is to be found in the loss of mathematical semantics. Current educational practice cares mostly to define variables or physical quantities and largely ignores the meaning of mathematical operations or relations. The practice resembles learning the grammar, syntax, of a foreign language without learning vocabulary. Although the use of mathematical structures from vector analysis, e.g., the gradient operator, the divergence operator, and the Gauss theorem, is essential for the very existence of transport theory, their meaning is overlooked mostly in calculus courses and transport courses alike.

Mathematics in engineering from a semiotic point of view is a code; it is a means of communicating ideas, created by humans and directed to humans. A code — a language is a codeconsists essentially of three components: syntax, rules or grammar; semantics, words or elements; and interpretation, what is conceived by a human when faced with a string of the code [4]. Interpretation requires context; a string of code can mean differently in different contexts. The three components are integral; we cannot, for instance, interpret a grammatically-sound English sentence, if we do not know the meaning of its words. ${ }^{1}$ A mathematical expression is essentially the same; it is a string of mathematical symbols; rules govern its structure; the symbols represent operations and variables; and potentially, it induces an interpretation in a certain context. We can,

\footnotetext{
${ }^{1}$ Deciphering the Rosetta Stone is a better example.
} 
then, interpret an equation, if we know both its structural rules and a meaning for each elementin a certain context; transport theory, for example. Otherwise, we cannot make sense of an equation.

A simple example best clarifies this thesis. When we say

$\varphi=\boldsymbol{\nabla} \cdot \mathbf{q}$

that a quantity $\varphi$ is the divergence of a quantity $\mathbf{q}$, we have expressed, not one category of information, but two. First, quantity $\varphi$ can be calculated from q using the technical, mathematical, definition of the divergence; this is the part that mathematics courses are mainly invested in. Indeed, a mathematics course will define the symbol del, $\boldsymbol{\nabla}$, in case of cartesian coordinates, as

$\boldsymbol{\nabla}=\frac{\partial}{\partial x} \hat{\mathbf{i}}+\frac{\partial}{\partial y} \hat{\mathbf{j}}+\frac{\partial}{\partial z} \hat{\mathbf{k}}$

Along with rules for differentiation, a typical mathematics class exhausts here its course of action regarding the first equation. It is all about the syntax, the techniques, the abstract relations between entities_-rules of an abstract game involving abstract players. ${ }^{2}$

The second category is the physical meaning, the well-known interpretation of that equation: the quantity $\varphi$ is the net outflow of quantity $\mathbf{q}$ at some point in space; and consequently, $\varphi$ is how much worth of $\mathbf{q}$ is generated (appears) or is destroyed (disappears) at that point. We have just appended the symbols with a meaning within the context of transport theory. In order to produce this interpretation, I had to attach a physical or geometrical meaning to the operation $[\nabla \cdot]$ on the quantity q; that is, I had to utilize a semantic system to all the elements of the equation. I call this command to distinguish it from technique. That could have not been possible in mathematics courses because the terms of flow, generation, destruction have not yet existed as context. Thus, the context for elucidating a meaning for a mathematical expression becomes available, not in calculus classes, but in later engineering courses; in our case, in a transport theory course. Physical terms, like field and flow, for this matter, do not coherently materialize in the minds of the students prior to teaching of mathematics courses in an engineering curriculum - these concepts are precisely the subject of subsequent theory courses. In sum, there is not much information or physical concepts that can be related or organized by the mathematical structures being taught at that time.

In this manner, mathematics is taught traditionally as techniques [5]. Afterwards, engineering theory courses in general assume a sufficient knowledge of both the mathematical technique and command. Theory courses operate and require an interpretative ability, an ability to abstract and mathematically analyze concrete phenomena, and inversely, to create mathematically-expressed ideas. Thus, an interface between the modes of a theory course (in a transport theory course) and mathematics coursework is missed, potentially making a consistent view of analysis of physical concepts difficult, even for students with fondness for mathematics.

\footnotetext{
${ }^{2}$ It is worth mentioning here that in Arabic and Persian, languages in which many contributions to mathematics were introduced, the word for mathematics is akin to terms of sports or gymnastics - a concept of mental gymnastics.
} 
The lack of command, further, confines the work of theory courses largely within the knowledge and application levels of Bloom's taxonomy [6], and severely inhibits all other levels for a majority of students. Command, as defined above, grants a fluency to involve all remaining levels of the taxonomy.

Considering the above, the loss of mathematical semantics or command leads the students not to associate a value to the analytical aspect of such courses. The students, in most cases, are exposed to diffusion, fluid flow or heat transfer in introductory physics classes. This implies that the value, and the purpose, of transport courses is exactly to add the analytical/theoretical layer on what they already perceive. Failure to grasp this value results in lack of fulfillment or appreciation to the course or the instructor, and, ultimately, in creating a psychological barrier and a student opinion against such courses.

\section{Solution strategy}

Addressing the above difficulties can only be done within theory courses, e.g., transport theory. This is due, as indicated, to the lack of context in mathematics courses to address the cause. A typical engineering student is initially founded in a series of mathematics courses intended to serve higher level proper engineering courses. Thus, by the very sequence of the course series, the context, for whose sake mathematics is taught (higher level engineering courses, e.g., transport or fluid mechanics courses) is inversely correlated to the method (mathematics courses in this case). Accordingly, the strategy is simply to provide the missing interface defined above: to dedicate time for explaining the meaning of mathematical operations after laying out an initial form of the theory concepts - in this case, the concepts of conservation of mass, energy, and momentum. It is argued that time spent on imparting meaning to mathematical logic and operations is of more learning value than the time currently spent on solution of advanced problems through calculation and evaluation (the drilling paradigm). The interface learning objectives are proposed as follows. Students will

- Attach a meaning to mathematical equations and operations (relevant to the course) and explain them in their own words, variables and operations alike; translate relevant mathematical expressions into plain language.

- Express plain language statements in mathematical form; formulate laws involving each of the conserved quantities, i.e., mass, energy and momentum, considering the physical nature of each quantity, i.e. scalar, vector or tensor.

The mathematical expressions should involve vector calculus operations, as relevant to transport theory. It is a good practice to use expressions involving gradient, divergence, curl, and combinations thereof using arbitrary variables. Achieving the above objectives should enable the students to construct fundamental and constitutive laws of transport themselves.

Such an interface possesses characteristics of threshold concepts: transformative, it changes the way the content is conceived; troublesome, its loss amounts to a conceptual inconsistency; irreversible, students will find difficulty in reverting back to meaningless operations; integrative, it creates a unifying theme that links the general laws with the method through to the application; discursive, being a semantic element expands the used language (Meyer and Land, 2003). 
The outlined learning objectives induce assessments that differentiate between two competencies to assess within the subject course: comprehension and formulation, on one hand, and evaluation, on the other hand. These two skills are fundamentally different with respect to the usual form of assessment, namely, problem solving in exams and homework. Students' ability to evaluate, i.e., work out formulae and derivations, is put on hold until they can sufficiently formulate and articulate transport problems in mathematical language. In alignment with the interface learning objectives, assignments are designed to assess the students' ability to comprehend and express in mathematical language. This practice paves the way for teaching an algorithmic approach to problem solving. An algorithmic approach requires articulation of (mathematical) steps without evaluations or calculations. Such approach can naturally create a motivation to engage in and solve problems which is mostly depressed for students who do not have natural fondness for mathematics as a practice. Meaning helps create a goal for abstract mathematical steps in the same manner applications motivate engineering problem solving [7].

\section{Pedagogy}

A course on transport theory consists, essentially, of a theory part and a methods part, the theory being on physical concepts and laws, and the methods being the mathematical structure in which to organize the content and implement it. Consequently, the big picture of the proposed pedagogy is to ground the course in the conceptual, theoretical, and required method fundamentals. It is seen that a foundation phase be enacted at the beginning of a transport theory course. This is on the basis that elucidating theoretical and methodological difficulties should pave a rational and successful way through the rest of the course content, making possible an inductive pedagogy [8].

A transport course which includes the three common topics of mass transfer, heat transfer, and fluid dynamics, matches an inductive approach. I see that the three components are symmetric; they are variations of the same idea of a conservation law and employ similar methods with varying complexity. Thus, an inductive approach starts from a foundation, utilizes that for heat or mass transfer and then advances to fluid dynamics. Ascending in complexity, the students are repeatedly familiarized with constructing transport models using similar methods. Topics such as fluid dynamics are really a sophisticated macrocosm of the basic concepts and methods. Importantly, the first sets of assessments are to focus on meaning of concepts as well as mathematical operations, command, in a way that excludes completely any evaluation exercise (e.g., boundary value problems).

To implement this, the proposed pedagogy is in the form of episodes of conceptual foundation intertwined with methods foundation. The first episode can be dedicated to a broad definition of the theory of transport and how it manifests itself in almost every aspect of natural and engineering systems.

The second episode delves into the concepts in which the physics of transport are cast. This ingrains the symmetry of such concepts for each of mass, energy, and momentum transfer. This episode may use the thermodynamic classification of intensive and extensive quantities. Each intensive quantity drives (and is driven by) a corresponding extensive quantity. An example of this is the pressure and flow rate. A definition of each intensive quantity should be introduced in tandem with the corresponding quantity. The discourse at this stage should converge to a conceptual 
framework that abstracts transport phenomena. The framework must organize quantities like pressure, flow rate and work for fluid movement; the chemical potential and concentration for mass transport; and temperature and entropy or heat for energy transport. The central purpose is to show the symmetry of concepts: for example, the pressure reflects a "concentration" of the momentum at a point in space; this is similar to the temperature as the "concentration" of thermal energy. Students are expected to see, through the different terminology, a unifying theme.

The third episode is an intermission to serve the molecular basis of transport phenomena. The symmetry of the concepts outlined above indicates a unifying underlying nature. This nature is precisely due to molecular constituents whether we speak about mass, energy or momentum transfer or all of them. Here, the content of the previous episode will be portraited as emergent properties from the molecular behavior. The kinetic theory of gases and Einstein's theory of Brownian motion will be discussed as examples of how macroscopic properties emerge from molecular interactions. Historical context will be mentioned to highlight how the macroscopic view preceded (and motivated) the microscopic view. Simulations can be used to show how diffusion occurs at the molecular level and how the same system, when zoomed out from, appears as a different quantity: that of a concentration field. This episode aims at addressing the misconceptions of emergent and direct causalities [9], [10].

The fourth episode is the statement of the idea of conservation. Given a knowledge of macroscopic quantities, students will be able to parse the quantities required to enact a conservation of mass, energy and momentum. It should be emphasized that this is the gist of transport theory and that what is done henceforth is directly traceable to this idea.

The fifth episode is the implementation of the first interface learning objective: attaching meaning to relevant mathematical operations. This focuses on the three fundamental operators from vector calculus: the gradient, the divergence and the curl and demonstrates what they physically or geometrically mean. Physical models should be used to translate such operations concretely. Mathematical statements with a combination of such operations should be read and translated into plain language.

The final episode of the foundation phase is to espouse the above concepts and methods where the students will be guided to formulate the general conservation laws in mathematical terms (the second learning objective above). At this stage, the students should be able to write such laws themselves in mathematical form, translating physical statements to mathematical form. This concludes the foundation phase where the two interface learning objectives are achieved. The second phase is then dedicated to projecting the outlined foundation to the details of mass, energy, and momentum transport relevant to the desired engineering discipline.

\section{Acknowledgments}

I am deeply grateful to Professor Ruth Streveler for her guidance and support. The ideas in this paper materialized within a course taught by Professor Streveler in Spring 2017.

\section{References}


[1] C. S. Dweck, Self-theories: Their Role in Motivation, Personality, and Development. Philadelphia: Psychology Press, 1999.

[2] A. Rattan, C. Good, and C. S. Dweck, "'It's ok - Not everyone can be good at math': Instructors with an entity theory comfort (and demotivate) students," J. Exp. Soc. Psychol., vol. 48, no. 3, pp. 731-737, 2012.

[3] A. H. Schoenfeld, "Purposes and Methods of Research in Mathematics Education," Not. AMS (American Math. Soc., vol. 47, no. 6, pp. 641-649, 2000.

[4] U. Eco, A Theory of Semiotics, 1st ed. Bloomington, Indiana: Indiana University Press, 1979.

[5] A. H. Schoenfeld, "Problem solving in the United States, 1970-2008: research and theory, practice and politics," ZDM Math. Educ., vol. 39, no. 5-6, pp. 537-551, 2007.

[6] D. R. Krathwohl, “A Revision of Bloom's Taxonomy: An Overview," Theory Pract., vol. 41, no. 4, pp. 212-218, 2002.

[7] M. D. Svinicki, Learning and Motivation in the Postsecondary Classroom. Jossey-Bass, 2004.

[8] P. C. Wankat and F. S. Oreovicz, "Teaching Engineering," in Teaching Engineering, 2nd ed., New York: McGraw-Hill, 2014.

[9] M. T. H. Chi, R. D. Roscoe, J. D. Slotta, M. Roy, and C. C. Chase, "Misconceived causal explanations for emergent processes," Cogn. Sci., vol. 36, no. 1, pp. 1-61, 2012.

[10] R. L. Miller, R. A. Streveler, D. Yang, and Aidsa I. Santiago Román, "Identifying and repairing student misconceptions in thermal and transport science: Concept inventories and schema training studies," Chem. Eng. Educ., vol. 45, pp. 203-210, 2011. 